\title{
Nanocrystalline Ge films created by thermal vacuum deposition on GaAs substrates: structural and electric properties
}

\author{
V.L. Borblik*, A.A. Korchevoi, A.S. Nikolenko, V.V. Strelchuk, A.M. Fonkich, \\ Yu.M. Shwarts, M.M. Shwarts \\ V. Lashkaryov Institute of Semiconductor Physics, NAS of Ukraine, \\ 41, prospect Nauky, 03028 Kyiv, Ukraine \\ ${ }^{*}$ Corresponding author e-mail: borblik@isp.kiev.ua
}

\begin{abstract}
The technique of thermal vacuum deposition of Ge onto GaAs substrates has been used for obtaining nanocrystalline Ge films. Nanocrystalline character of the films is confirmed by atomic force microscopy of their surface and by the data of Raman light scattering. The most probable size of the nanocrystallites forming the films decreases monotonically with decreasing their thickness. Electro conductivity of such the films proves to be high enough $(1-10 \mathrm{Ohm} \cdot \mathrm{cm}$ at room temperature) and has a character of variable range hopping conduction of Mott's type. The hops, presumably, take place through the localized states connected with the grain boundaries.
\end{abstract}

Keywords: thermal vacuum deposition, germanium, gallium arsenide, nanocrystalline films, hopping conduction.

Manuscript received 07.02.14; revised version received 25.06.14; accepted for publication 16.09.14; published online 30.09.14.

\section{Introduction}

The first works concerning with germanium epitaxy on gallium arsenide substrate (in 60-ies) were carried out by means of thermal deposition in vacuum and had not satisfied researchers in many respects because they had failed to get the films with electric properties close to those of bulk Ge.

Since then the variety of more complete technique have been developed (molecular beam epitaxy, chemical-vapor deposition, high-vacuum magnetron sputtering et al.) that have resulted in refining such kind of films up to so-called "device quality" which is characterized by high carrier mobility and large enough lifetimes of non-equilibrium carriers, i. e. by high crystalline perfection (see, for example, [1]).

At the same time a new science direction has appeared (nanophysics) in the framework of which non- perfect crystallinity, more exactly - nanocrystallinity, is just desirable property. Wide investigation of nanocrystalline (or nanostructured) objects is connected with significant changes of all properties of the materials (mechanical, electrical, optical) in nanostructured state $[2,3]$. This circumstance allows returning to simple and cheap technology of thermal vacuum deposition.

Nanostructured state of Ge film is attractive, uppermost, in connection with the possibility to obtain a luminescence in visible range of the spectrum. It is important also that these films should have a high enough electro conduction that will allow obtaining the active light emitting devices on their base.

In the course of present investigations, the different regimes of $\mathrm{Ge}$ deposition on GaAs substrate have been studied and those of them which result in nanocrystalline films have been found. Then structural and electric properties of the films obtained have been studied. 


\section{Experimental}

The crucible made of tantalum-graphite which was heated by alternate current up to temperature of $\sim 1400$ $1600{ }^{\circ} \mathrm{C}$ and derived thermal evaporation of $\mathrm{Ge}$, was located in vacuum camera with pressure of $(2-5) \cdot 10^{-6}$ Torr. Temperature of the (100)GaAs substrate with resistivity of $10^{8} \mathrm{Ohm} \cdot \mathrm{cm}$ could be supported in the range of $200-550^{\circ} \mathrm{C}$ by the special heater.

The film thickness was measured by means of micro profilometer "Alpha-Step 100" with accuracy of $4 \mathrm{~nm}$ and by micro interferometer MII- 4 .

Measurements of micro Raman light scattering were carried out in reflection geometry at room temperature by means of triple Raman spectrometer T64000 Horiba Jobin-Yvon equipped with cooled CCD detector. For the sample excitation, light from $\mathrm{Ar}-\mathrm{Kr}$ ionic laser with wavelength of $488 \mathrm{~nm}$ are focused to the sample by objective $50 \times / 0.75$ to the spot of $\sim 1 \mu \mathrm{m}$ size under the radiation power near $1 \mathrm{~mW}$.

Investigations of the film surface were carried out by atomic force microscope NanoScope IIIa Dimention 3000 (Digital Instruments, CШA) in the regime of periodic contact. The serial silicon probes NSG-11 with nominal tip curvature radius of $10 \mathrm{~nm}$ (NT-MDT, Russia) were used.

For measuring conductive properties of the films obtained, planar contacts with the spacing of $1 \mathrm{~mm}$ were deposited on them.

\section{Results and discussion}

\subsection{Technological conditions for nanocrystallinity of the films}

Fig. 1 represents the data of Raman light scattering for three films: $\# 2$ and $\# 3$ - deposited at temperature of 500 and $350{ }^{\circ} \mathrm{C}$, respectively, on the substrates annealed at $500{ }^{\circ} \mathrm{C}$ for $20 \mathrm{~min}$, and $\# 4$ - deposited at temperature of $300^{\circ} \mathrm{C}$ on non-annealed substrate.

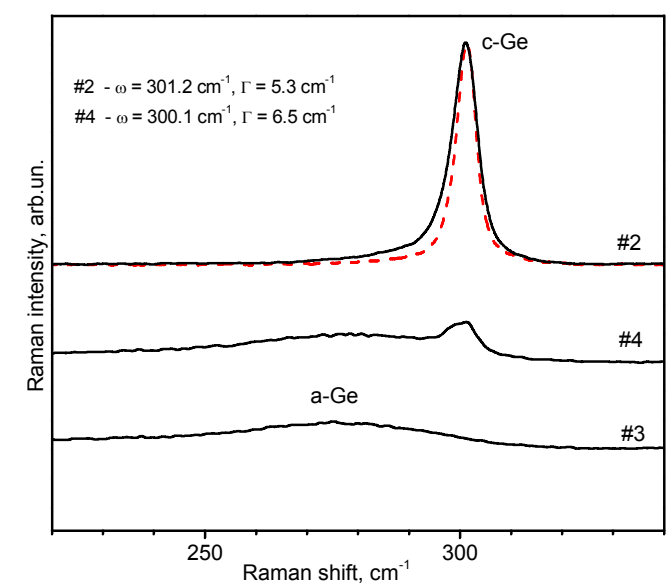

Fig. 1. Spectra of Raman light scattering for the samples \#2, \#3 and \#4; dashed curve represents spectrum of bulk Ge.
At low deposition temperature $\left(350^{\circ} \mathrm{C}\right)$, Ge film turns out amorphous as it follows from style of the curve for sample \#3 in the form of diffuse peak. But at deposition temperature of $500{ }^{\circ} \mathrm{C}$ (sample \#2) the sharp peak appears near the optic phonon frequency of $300 \mathrm{~cm}^{-1}$ which corresponds to crystalline Ge. However this peak is broadened in comparison with that in the bulk material (dashed curve in Fig. 1) due to polycrystalline (grain) structure of the obtained film and is asymmetrical because of the effect of quantum confinement for phonons in the nano-dimensional crystallites. Also the peak maximum undergoes the shift to lower frequencies. And the film deposited at $300^{\circ} \mathrm{C}$ on non-annealed substrate (sample \#4) turns out amorphous as well (though with some portion of crystalline phase).

\subsection{Structure of the films surfaces}

Results of the atomic force microscopy (AFM) are represented in Fig. 2 for a number of the films (obtained by $\mathrm{Ge}$ deposition at temperature of $380{ }^{\circ} \mathrm{C}$ on the substrates annealed previously at $400{ }^{\circ} \mathrm{C}$ for $8 \mathrm{~min}$ ) which have different thicknesses $-450,150$ and $75 \mathrm{~nm}$. Fig. 3 represents analogous data for even more thin film with thickness of $15 \mathrm{~nm}$ obtained under somewhat another conditions - deposition at $400{ }^{\circ} \mathrm{C}$ on the nonannealed substrate.

All of them have a grain, polycrystalline, structure. But characteristic grain sizes turn out different depending on the film thickness. Distribution of the effective lateral grain sizes is presented on the corresponding histograms in Figs 2 and 3, and distribution of the vertical scales of the surface relief is presented in Fig. 4 (along with the results of their processing as the gauss curves).

As it follows from the data represented, decrease in the film thickness (from 450 to $15 \mathrm{~nm}$ ) results in decreasing all characteristic scales of their surface relief. So the most probable lateral grain size decreases from $40-60 \mathrm{~nm}$ through $35-40 \mathrm{~nm}$ (then $30-35 \mathrm{~nm}$ ) down to 24-26 nm. The most probable value of the relief height decreases from $19.26 \mathrm{~nm}$ through $11.68 \mathrm{~nm}$ (then $7.98 \mathrm{~nm}$ ) down to $3.97 \mathrm{~nm}$, and the root-mean-square height deviation from the average value (roughness) from $5.73 \mathrm{~nm}$ through $3.3 \mathrm{~nm}$ (then $2.60 \mathrm{~nm}$ ) down to $1.18 \mathrm{~nm}$. It is obvious that gain of the grain size with increase in the film thickness is connected directly with increase in the time spent for their growth that results in enlargement of the crystallites.

It is interesting that qualitatively similar dependence between film thickness and grain size has been observed in the case of hydrogenised germanium films on glass [4]. As was noted in Ref. [4] the values obtained by means of AFM prove to be somewhat overrated because of the finite tip radius; therefore the lateral scale of the film nanostructurization can turn out even smaller in fact. 
Above obtained dependences of the characteristic scales of the surface relief on the film thickness are summarized in Fig. 5. It is worthwhile to pay attention to practically complete identity of the thickness dependences of the characteristic scales for lower boundary of the lateral sizes of the grains (a, bottom) and their characteristic height (b).

In all the cases a ratio of the double characteristic height to the characteristic lateral size is less than 1, i.e. the grains (at least, in the near-surface layer) are oblate in direction perpendicular to the surface, and their oblateness increases with decrease in the film thickness. In Fig. 6 this fact is demonstrated for the thinnest film $(15 \mathrm{~nm})$ where the height of each element of the film surface relief is plotted versus its effective lateral size. It should be noted that authors of paper [5] also took notice an oblateness of the grains on the surface of the films consisting of $\mathrm{Ge}$ nanocrystals embedded in $\mathrm{SiO}_{2}$ matrix.

\subsection{Conductive properties of the films}

All the obtained films had linear current-voltage characteristics up to applied voltage of $10 \mathrm{~V}$ in the temperature range of $77-300 \mathrm{~K}$.

Fig. 7 represents Arrenius curves for the current $I$ at the fixed voltage $U=1 \mathrm{~V}$ for two such the films: \#13 (the film with thickness of $150 \mathrm{~nm}$ deposited at temperature of $400{ }^{\circ} \mathrm{C}$ on the substrate annealed previously at $450^{\circ} \mathrm{C}$ for $5 \mathrm{~min}$ ) and \#16 (the film with the same thickness of $150 \mathrm{~nm}$ deposited at temperature
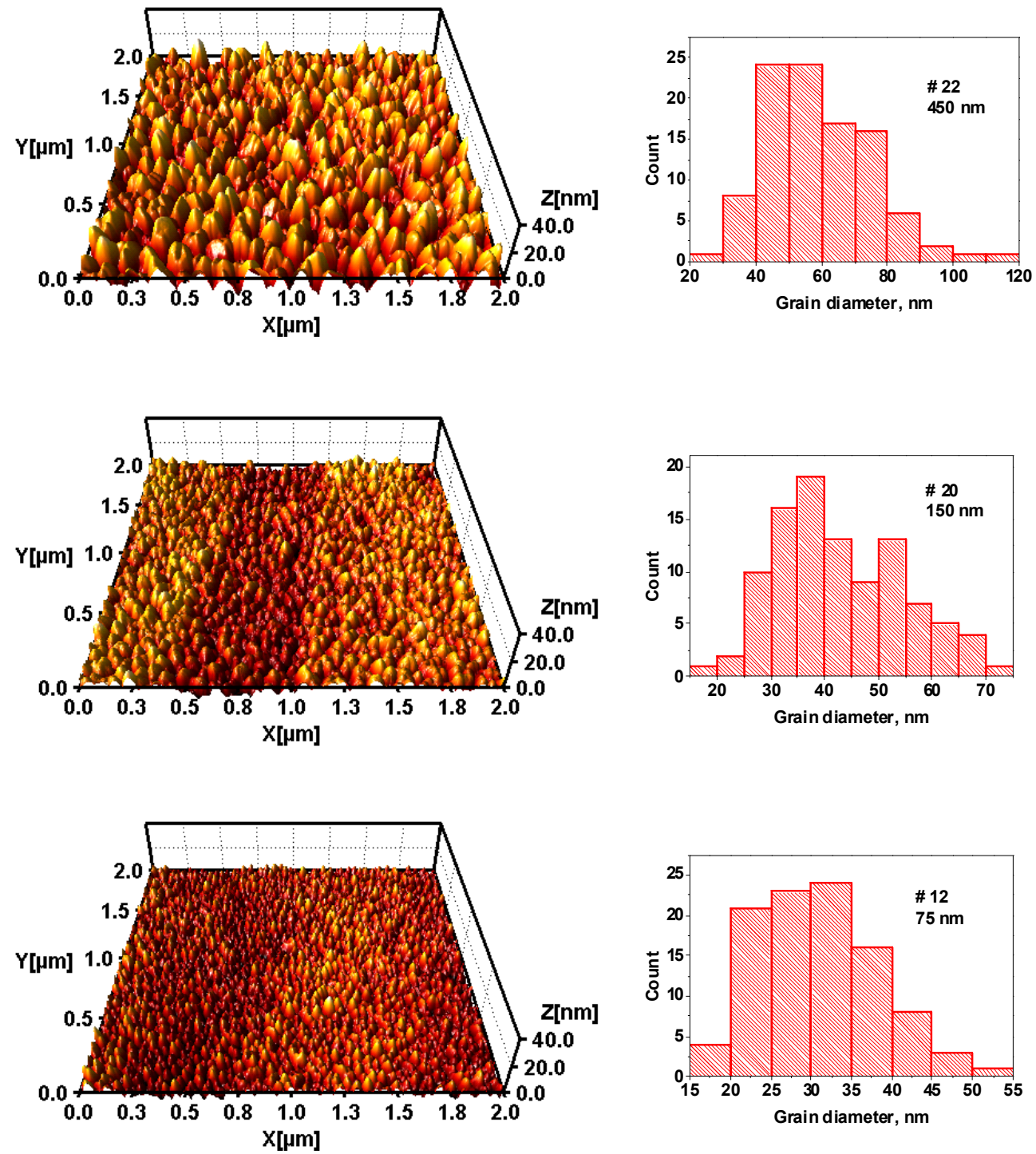

Fig. 2. Images of the film surface in atomic force microscope and histograms of the lateral grain sizes for Ge films with the thickness of $450(a), 150(b)$ and $75 \mathrm{~nm}(c)$ on the GaAs substrates.

(C) 2014, V. Lashkaryov Institute of Semiconductor Physics, National Academy of Sciences of Ukraine 

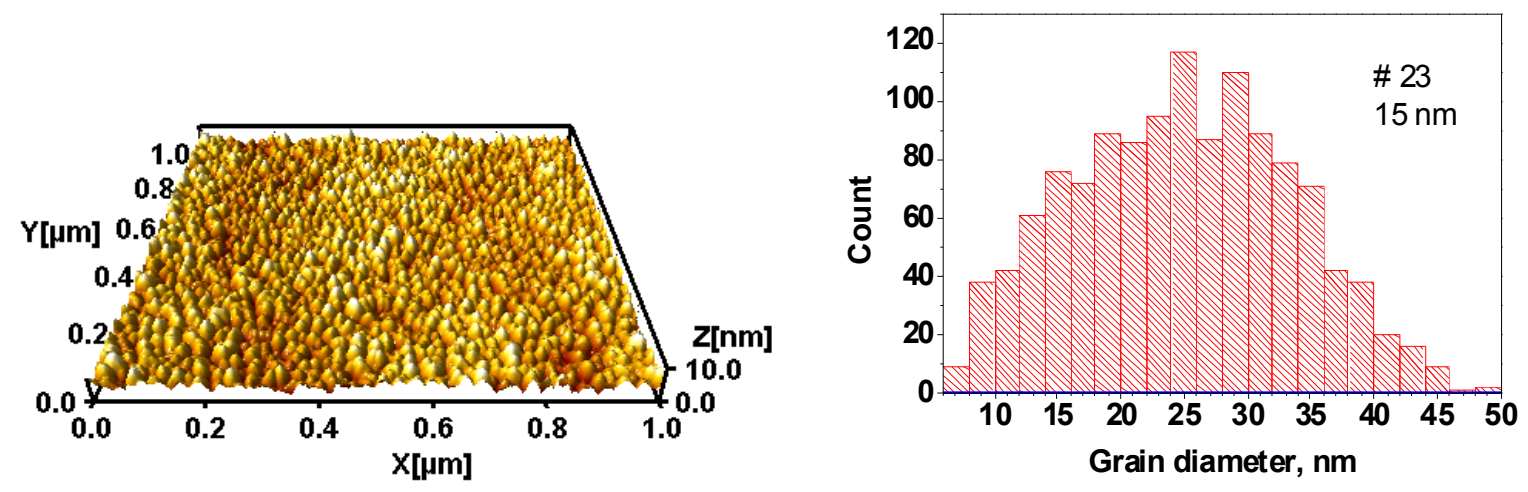

Fig. 3. The same as in Fig. 2 for the Ge film with the thickness of $15 \mathrm{~nm}$ on the GaAs substrate.

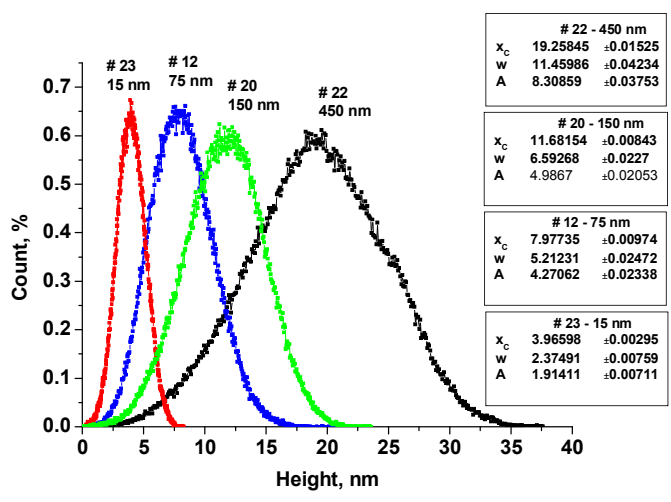

Fig. 4. Distribution of the height probabilities for the surface relief of the films shown in Figs 2 and 3 along with results of the data approximation by the gauss curves (by means of program Origin).

of $380{ }^{\circ} \mathrm{C}$ on the substrate annealed previously at $400^{\circ} \mathrm{C}$ for $6 \mathrm{~min}$ ). These curves determine temperature dependences of the film electro conductivity $\sigma$ which (as it is seen from the figure) is characterized by the variable activation energy. These dependences are straightened well being plotted as a function of $(1 / T)^{1 / 4}$ as it is demonstrated by Fig. 8. It follows from this that the film conductivity is realized by means of variable range hopping conduction of Mott's type when $\ln \sigma \propto\left(T_{0} / T\right)^{1 / 4}$. Here $T_{0}=\frac{\text { const }}{\operatorname{kg}\left(E_{\mathrm{F}}\right) a}$ where $k$ is the Boltzmann constant, $a$ is the localization radius of the states through which the hops take place, $g\left(E_{\mathrm{F}}\right)$ is the density of these states on the Fermi level. Values of parameter $T_{0}$ determined from the line slope for two samples differ noticeably from each other.

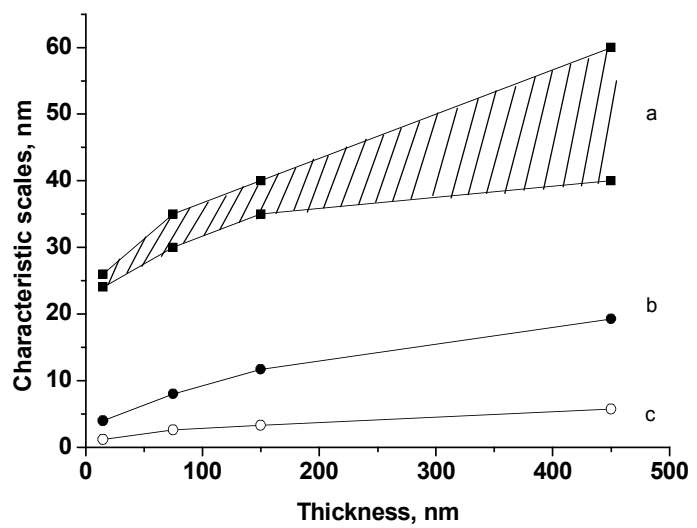

Fig. 5. Dependences of the characteristic lateral nonhomogeneity scale of the film surface $(a)$, the characteristic scale of the relief height $(b)$ and the root-mean-square deviation of the height from average value (roughness) $(c)$ on the film thickness.

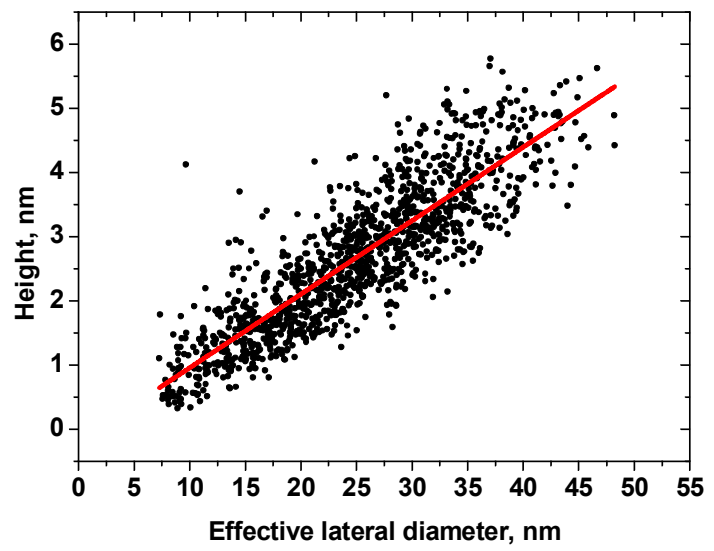

Fig. 6. Dependence between the heights of the surface relief elements and their effective lateral sizes for the film with the thickness $15 \mathrm{~nm}$ (the sample \#23). 
It is worthwhile to look for a reason for significant difference in the conductive properties of these two films in their morphology. Shown in Figs 9 and 10 are the data of the AFM of their surfaces.

It follows from these figures that the film \#13 consists of much larger crystallites than the film \#16. It is obvious also that the distances between crystallites will be more substantial in the film \#13 than in the film \#16 that can explain its significantly smaller conductivity.

From comparison of the characteristic temperatures $T_{0}$, it follows that the film \#13 has larger density of

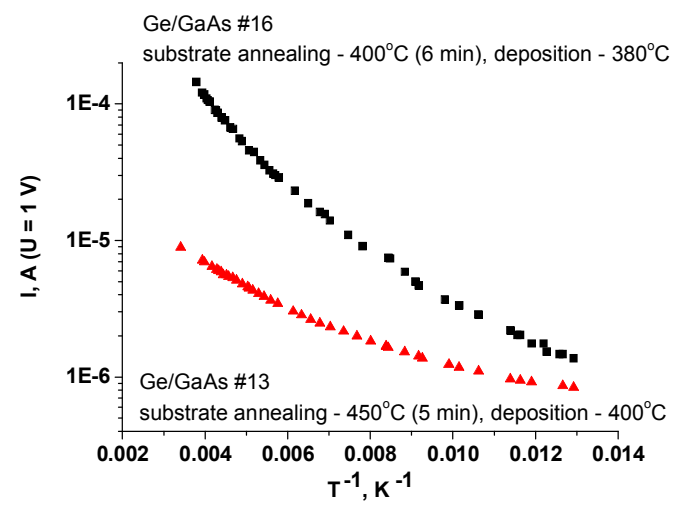

Fig. 7. Arrenius curves for the current along the film at fixed voltage of $1 \mathrm{~V}$ for the samples \#13 and \#16.
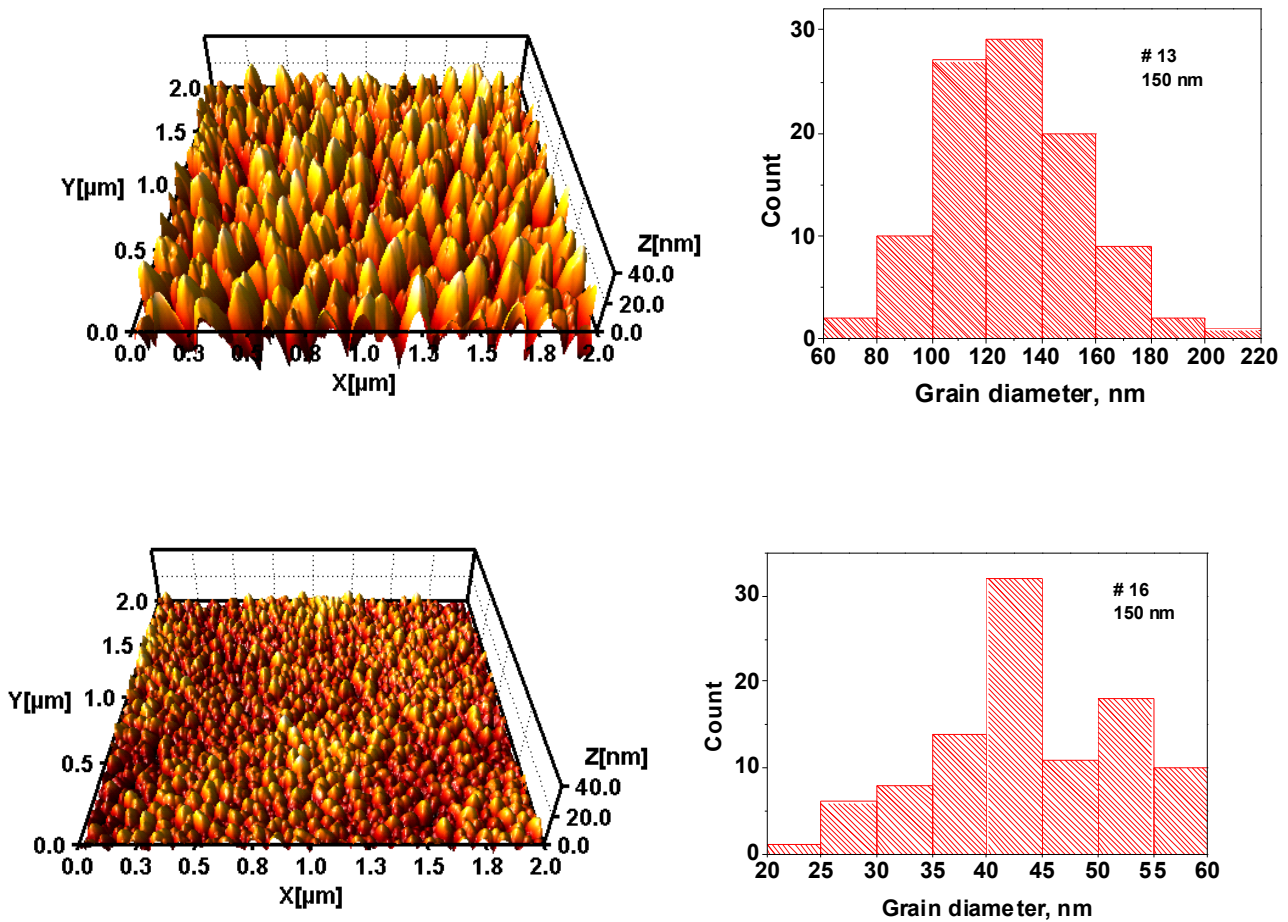

b

Fig. 9. Images of the film surface in atomic force microscope and histograms of the lateral grain sizes for the samples \#13 (a) and $\# 16$ (b). 


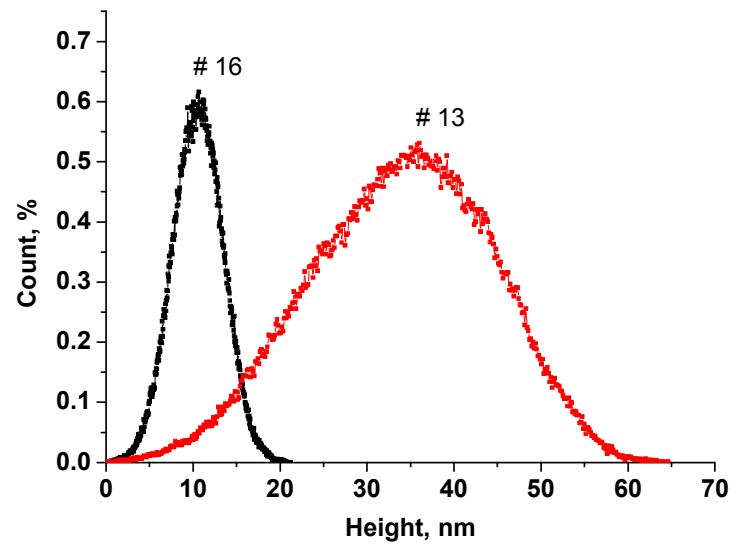

Fig. 10. Distribution of the height probabilities for the surface relief of the samples \#13 and \#16.

Authors of this paper have observed decrease in $T_{0}$ with the growth of Ge content in the films, i.e. with increase in either sizes or number of conductive clusters that results in increasing $g\left(E_{\mathrm{F}}\right)$.

Note that in the case when $\mathrm{Ge}$ in $\mathrm{SiO}_{2}$ matrix has been transformed into nanocrystalline inclusions with sizes of 4-9 $\mathrm{nm}$ [7] (as a result of following thermotreatment), the same authors have observed the temperature dependence of the film conductivity in the form of $\ln \sigma \propto(1 / T)^{1 / 2}$. In spite of similarity of this dependence with that for variable range hopping conduction under coulomb gap [8], this mechanism is not considered as appropriate to given situation.

The temperature dependence in the form of $\ln \sigma \propto\left(T_{0} / T\right)^{1 / 4}$ has been observed also in the paper [9] for the conductivity along the films of similar type (Ge nanoparticles embedded in $\mathrm{SiO}_{2}$ matrix) and for the conductivity across the films which consist of $\mathrm{Ge}$ nanocrystals on silicon or silica substrates grown at room temperature [10]. In all similar cases, the variable range hopping conduction of Mott's type is explained by the existence of the localized states connected with the grain boundaries.

\section{Conclusions}

Thus the high enough temperature of the GaAs substrate under deposition of Ge film (above $380-400{ }^{\circ} \mathrm{C}$ ) is the condition of obtaining its nanocrystalline structure. The thinner the film, the smaller are the crystallites forming it; this allows us to choose purposefully their desirable size (at the average). In view of smallness of the distances between the adjacent nanograins in the films, their conductivity proves to be high enough (1$10 \mathrm{Ohm} \cdot \mathrm{cm}$ at room temperature) that will allow creation of active light emitting devices on the base of such the films.

\section{References}

1. S.-H. Tang, E. Y. Chang, M. Hudait, J.-S. Maa, C.-W. Liu, G.-L. Luo, H.-D. Trinh, and Y.-H. Su, High quality Ge thin film grown by ultrahigh vacuum chemical vapor deposition on GaAs substrate // Appl. Phys. Lett. 98(16), 161905(1-3) (2011).

2. H. Gleiter, Nanostructured materials: basic concepts and microstructure // Acta Materialia, 48(1), p. 1-29 (2000).

3. R.A. Andrievskii and A.V. Ragulya, Nanostructured Materials. Academia, Moscow, 2005.

4. W.B. Jordan, E.D. Carlson, T.R. Johnson, and S. Wagner // Mat. Res. Soc. Symp. Proc. 762, paper A6.5 (2003).

5. I. Stavarache, A.-M. Lepadatu, T. Stoica, and M.L. Ciurea, Annealing temperature effect on structure and electrical properties of films formed of $\mathrm{Ge}$ nanoparticles in $\mathrm{SiO}_{2} / /$ Appl. Surf. Sci. 285, Part B, p. 175-179 (2013).

6. M. Fujii, Y. Inoue, S. Hayashi, and K. Yamamoto, Hopping conduction in $\mathrm{SiO}_{2}$ films containing $\mathrm{C}, \mathrm{Si}$, and Ge clusters // Appl. Phys. Lett. 68(26), p. 37493751 (1996).

7. M. Fujii, O. Mamezaki, S. Hayashi, and K. Yamamoto, Current transport properties of $\mathrm{SiO}_{2}$ films containing Ge nanocrystals // J. Appl. Phys. 83(3), p. 1507-1512 (1998).

8. B.I. Shklovskii and A.L. Efros, The Electronic Properties of Doped Semiconductors. Nauka, Moscow, 1979.

9. I. Stavarache, A.-M. Lepadatu, A.V. Maraloiu, V.S. Teodorescu, and M.L. Ciurea, Structure and electrical transport in films of Ge nanoparticles embedded in $\mathrm{SiO}_{2}$ matrix // J. Nanopart. Res. 14(7), Article: 930(1-9) (2012).

10. S. Banerjee, Non-ohmic hopping conduction in Ge nanocrystalline film // Physica E, 15(3), p. 164-168 (2002). 